\title{
Isozyme Analysis of Taenia solium Isolates from Mexico and Colombia
}

\author{
Pablo Maravilla/* , Aldo Valera**, Valeria Souza**, Mario Martinez-Gordillo***, \\ Ana Flisser/*/+
}

Hospital General “Dr. Manuel Gea Gonzalez”, 14400 México DF, México *Facultad de Medicina **Instituto de Ecología,
UNAM, México ***Instituto Nacional de Pediatria, México DF, México

Mexican and Colombian Taenia solium cysticerci and some species of Taenia adults were assayed using cellulose acetate electrophoresis to distinguish between isolates. Isozyme patterns for ARK, GOT, G3PD, GPI, and MPI were identical in all cysticerci suggesting homozygotic profiles. G6PD and MDH showed different patterns between Mexican and Colombian cysticerci, suggesting regional differences. ME activity was mainly detected in the adult stage suggesting that this enzyme is active in anaerobic environment, while $M D H$, detected in cysticerci, could be related to an environment that contains oxygen. Finally, the species of taeniid adults analyzed showed different patterns among them.

Key words: Taenia solium - isozymes - cysticerci - Mexico - Colombia

Taeniosis and cysticercosis are two diseases caused by the cestode Taenia solium. Thompson and Lymbery (1995) argue that knowledge of genetic structure of cestodes can be applied to the epidemiology and the control of these parasites, because genetic variation within and between populations, determines future evolutionary changes, genetic differentiation, and speciation. In order to distinguish within isolates from $T$. solium, isozyme electrophoresis analysis was performed in parasites recovered from Mexico and Colombia, and in adult tapeworms.

Ten $T$. solium cysticerci were excised from each of seven naturally infected pigs from central states of Mexico and one pig from Colombia, parasites were washed with $0.01 \mathrm{M}$ phosphate-buffered $0.15 \mathrm{M}$ saline, $\mathrm{pH} 7.2$ (PBS), dried over filter paper, frozen $\left(-70^{\circ} \mathrm{C}\right)$ and individually analyzed for isozymes. Mexican immature proglottids of $T$. solium and $T$. saginata adult worms from human hosts recovered prior to cestocidal treatment were frozen and similarly analyzed. Immature proglottids from Taenia taeniaeformis recovered from experimental cat infections were also used. Cellulose acetate (CA) electrophoresis of enzymes was conducted according to the methods of Hebert and Beaton (1993) and Johnston and Fernando (1997). Eight isoenzymes were tested: ARK, EC. 2.7.3.3; G6PD, EC. 1.1.1.49; GPI. EC. 1.2.1.12; G3PD, EC. 1.2.1.12;

Financial partial support: grant 28094-B from the Consejo Nacional de Ciencia y Tecnología and project 102344 from the Programa de Apoyo a Estudiantes de Posgrado.

${ }^{+}$Corresponding author. Fax: +52-55-5623.2382. E-mail: flisser@servidor.unam.mx

Received 14 July 2003

Accepted 19 November 2003
GOT, EC. 2.6.1.1; MDH, EC, 1.1.1.37; ME, EC. 1.1.1.40; MPI, EC. 5.3.1.8. Individual parasites were homogenized in an equal weight-volume ratio of distilled water and centrifuged in at $14,000 \mathrm{rpm}$ for $15-30 \mathrm{~s}$. Supernatants were chilled on ice and loaded onto Titan III 76x76 mm CA plates (Helena Laboratories, Beaumont, TX). Electrode buffers and electrophoresis conditions are outlined in the Table. One "hit" $(\sim 3 \mu \mathrm{l})$ per plate for each enzyme was delivered employing the Super Z-12 applicator kit (Helena Laboratories, Beaumont, TX). Soluble meat extracts from a healthy pig were used as control.

The electrophoretic mobility of G6PD, GPI, GOT, ARK, and G3PD from all cysticerci recovered from the isolates showed only one band of activity, MDH and ME (only for T. solium adult) showed 2 bands and MPI presented 3 bands making a total of 10 putative alleles. Enzyme mobility was the same for Mexican and Colombian cysticerci, except for GPI and MDH that were slightly faster with Colombian than Mexican cysticerci (Fig. 1A). Each Taenia species tapeworm tested showed its own enzyme pattern, being clearly distinct for GPI, ME (data not shown), and MPI (Fig. 1B). Activity of ME was observed in adult tapeworms but not in cysticerci, suggesting to be stage-specific. To confirm this observation, two other methods were used: isoelectrofocusing and spectrophotometry as described by Ponce-Macotela et al. (1993). The former confirmed this result, while the latter measured 4 times less activity in cysticerci than in adult parasites $(0.122$ vs $0.512 \mathrm{ab}-$ sorbance values at $570 \mathrm{~nm}$ using $20 \mathrm{mg} / \mathrm{ml}$ total protein in each extract), indicating that although there is some activity in cysticerci it is higher in adult worms. Isozyme profiles from cysticerci were different from those seen in pig meat (Fig. 1, lane 1) which confirms lack of meat contamination.

The trimeric pattern of GPI from T. solium, T. saginata, and T. taeniaeformis was according to profiles published by Le Riche and Sewell (1977) as well as Okamoto and 
TABLE

Electrophoresis conditions

\begin{tabular}{lccc}
\hline Enzyme & Tank buffer & Time (min) & Volts \\
\hline G6PD, MDH, G3PD & CAAPM $^{a}$ & 120 & 38 \\
GOT, GPI & TM $^{b}$ & 120 & 38 \\
ARK, ME, MPI & TM & 20 & 170 \\
\hline
\end{tabular}

$a$ : $0.22 \mathrm{M}$ citric acid, 5\% (v/v) 4-(3-amonipropyl) morpholine, $\mathrm{pH} 8.2 ; b$ : 0.25M TRIS, $0.05 \mathrm{M}$ malic acid, $\mathrm{pH} 7.8$
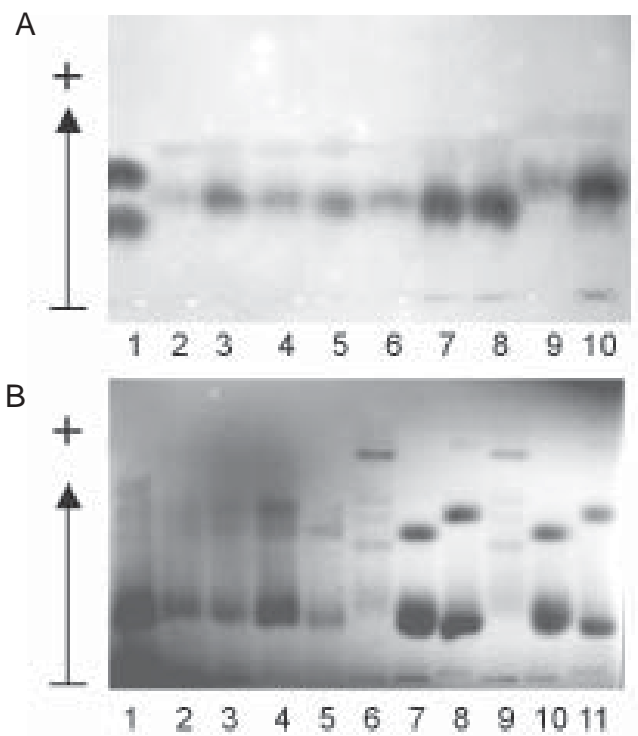

Fig. 1: isoenzyme patterns - A: MDH from Mexican cysticerci (lanes 2-8), and Colombian cysticerci (lanes 9-10); B: MPI from Mexican cysticerci (lanes 2-5) adult Taenia taeniaeformis (lanes 6 and 9), adult $T$. solium (lanes 7 and 10), and adult T. saginata (lanes 8 and 11). Swine muscle extract was analyzed in lanes 1 .

colleagues (1995). Housekeeping enzymes, such as GPI, therefore, confer a phenotypic identity to each parasite, and could be used for differential diagnosis with modern molecular methods. Various enzymes have been tested in order to learn about evolution ecology and population genetics of cestodes (Okamoto et al. 1995, Siles-Lucas et al. 1996, Turèeková et al. 2003). In the present study no differences in the migration of 5 isozyme profiles (ARK, GOT, G3PD, GPI, and MPI) were found suggesting homozygotic profiles. On the other hand, the mobility of G6PD and MDH was slightly faster in Colombian than in Mexican cysticerci suggesting regional differences that have to be confirmed with a larger sample of cysticerci from Colombia or from other countries. The presence of detectable ME activity in the adult stage can be explained because this enzyme has high activity in anaerobic environment (like the intestinal lumen), while $\mathrm{MDH}$, that was only detected in cysticerci, has better activity in an environment that contains oxygen (such as skeletal muscle, where the T. solium cysticerci develop); this feature could be exploited in studies focused for the treatment and control of this parasite.

\section{ACKNOWLEDGEMENTS}

To Agustin Plancarte (UNAM) for T. taeniaeformis material and to Dr Laura Vargas-Parada (Hospital Gea) for critical comment on the manuscript.

\section{REFERENCES}

Hebert PDN, Beaton MJ 1993. Methodologies for Allozyme Analysis Using Cellulose Acetate Electrophoresis: A Practical Handbook, Helena Laboratories, Beaumont, TX, $39 \mathrm{pp}$.

Johnston DA, Fernando MA 1997. Isoenzymes of Eimeria from the domestic fowl: electrophoretic variants among species, strains and clones. Parasitol Res 83: 464-470.

Le Riche PD, Sewell MMH 1977. Differentiation of Taenia saginata and Taenia solium by enzyme electrophoresis. Trans R Soc Trop Med Hyg 4: 327-328.

Okamoto M, Ito A, Kurosawa T, Oku Y, Kamiya M, Agatsuma $\mathrm{T}$ 1995. Intraspecific variation of isoenzymes in Taenia taeniaeformis. Int J Parasitol 25: 221-228.

Ponce-Macotela M, Martinez-Gordillo MN, Alvarez-Chacon R 1993. Giardia duodenalis: analysis of malic enzyme expression with isoelectric focusing. Rev Invest Clin (Mexico)45: 371-374.

Siles-Lucas M, Cesar-Benito M, Cuesta-Bandera C 1996. Echinococcus granulosus: genomic and isoenzymatic study of Spanish strains isolated from different intermediate hosts. Vet Parasitol 63: 273-282.

Thompson RCA, Lymbery AJ 1995. Echinococcus and Hydatid Disease, CAB International, Wallingford, UK, 477 pp.

Turèeková L, Šnábel V, D’Amelio S, Busi M, Dubinský P 2003. Morphological and genetic characterization of Echinococcus granulosus in the Slovak Republic. Acta Trop 85: 223229. 